\title{
Pancreatic cancer and immune checkpoint inhibitors-still a long way to go
}

\author{
Jessica Bian ${ }^{1}$, Khaldoun Almhanna ${ }^{2}$ \\ ${ }^{1}$ Division of Hematology/Medical Oncology, Maine Medical Center, MaineHealth Cancer Care, Portland, ME, USA; ${ }^{2}$ Division of Hematology/ \\ Oncology, The Warren Alpert Medical School of Brown University, Lifespan Cancer Institute, Rhode Island Hospital, Providence, RI, USA \\ Correspondence to: Jessica Bian, MD. Division of Hematology/Medical Oncology, Maine Medical Center, MaineHealth Cancer Care, Portland, ME \\ 04102, USA. Email: jbian@mmc.org.
}

Received: 15 November 2019; Accepted: 26 March 2020; Published: 05 January 2021.

doi: $10.21037 / \operatorname{tgh} .2020 .04 .03$

View this article at: http://dx.doi.org/10.21037/tgh.2020.04.03

Despite advances in immunotherapy in multiple solid tumor types, the role of immunotherapy in pancreatic ductal adenocarcinoma (PDAC) remains limited. PDAC has been reported to express a low tumor mutational burden and an immunosuppressive tumor microenvironment, both of which pose challenges to the success of immunotherapy for pancreatic cancer $(1,2)$. Earlier immunotherapy efforts in pancreatic cancer focused on vaccines, but thus far no large scale vaccine trials have demonstrated long-term efficacy. Notable completed pancreatic cancer vaccine studies include the TeloVac, IMPRESS, and ECLIPSE trials.

Telomerase is expressed in pancreatic cancer, and GV1001 is a telomerase peptide vaccine that demonstrated an immune response in phase I/II studies (3). TeloVac was an open-label, randomized, phase III trial evaluating the immunogenic telomerase peptide vaccine GV1001 in combination with gemcitabine and capecitabine in 1,062 patients with locally advanced or metastatic pancreatic cancer. Patients were randomly assigned 1:1:1 to receive chemotherapy alone, chemotherapy and sequential GV1001, or chemotherapy and concurrent GV1001. Median overall survival was 7.9 months with chemotherapy alone, 6.9 months with chemotherapy and sequential GV1001, and 8.4 months with chemotherapy and concurrent GV1001. The trial was terminated early in March 2011 due to decreased survival in the chemotherapy and sequential GV1001 groups (4).

IMPRESS was a phase III study of the pancreatic cancer vaccine algenpantucel-L in 722 patients with surgically resected pancreatic cancer. Algenpantucel- $\mathrm{L}$ is a vaccine comprised of allogeneic pancreatic cancer cells modified to express surface alpha-1,3-galactosyltransferase leading to hyperacute rejection of the vaccine allograft with a goal of inducing immune-mediated toxicity towards endogenous pancreatic cancer cells. Study patients received algenpantucel-L in combination with adjuvant gemcitabine with or without $5-\mathrm{FU}$ and radiation versus adjuvant gemcitabine with or without 5 -FU and radiation alone. Results were published in May 2016. The study did not reach its primary endpoint of improved overall survival; median overall survival was 30.4 months for the control group and 27.3 months for the study group (5).

A more recently completed pancreatic cancer vaccine trial was a phase IIb, randomized, multicenter study of GVAX pancreas and CRS-207 compared to singleagent chemotherapy in patients with previously treated metastatic pancreatic cancer (ECLIPSE). GVAX is an irradiated, autologous pancreatic cancer vaccine genetically modified to secrete granulocyte-macrophage colony stimulating factor with a goal of stimulating an immune response against pancreatic cancer cells. CRS-207 is a live, attenuated Listeria-based cancer vaccine expressing human mesothelin which can activate an immune response against mesothelin-overexpressing pancreatic cancer cells. ECLIPSE compared low-dose cyclophosphamide/GVAX/ CRS-207 (arm A) versus CRS-207 alone (arm B), or singleagent chemotherapy ( $\operatorname{arm} \mathrm{C}$ ). The study rationale for adding low-dose cyclophosphamide was to enhance the immune response by depleting regulatory $\mathrm{T}$ cells, based on previously published phase II data (6). Study subjects were divided into a primary cohort of patients who had received two or more lines of therapy (213 patients) and a 
second-line cohort of patients who had received one prior line of chemotherapy for metastatic disease (90 patients). The study did not meet the primary efficacy endpoint of improved overall survival of arm A compared to arm $\mathrm{C}$ in the intention-to-treat (ITT) primary cohort. The median overall survival in the primary cohort of arm A was 3.7 months, 5.4 months in arm B, and 4.6 months in arm C. It should be noted that there was a disproportionate dropout rate in arm $\mathrm{C}$ in both primary and secondary cohorts although there was not a significant difference in overall survival between the ITT and full analysis set populations (7).

Attention turned to immune checkpoint blockade following the disappointing results of pancreatic cancer vaccine trials. The anti-programmed death-1 (PD-1) immune checkpoint inhibitor pembrolizumab is the only immunotherapy that is FDA-approved for the treatment of patients with advanced PDAC-provided PDAC is mismatch repair deficient (dMMR) or microsatellite instability high (MSI-H). In May 2017, pembrolizumab was approved for patients with unresectable or metastatic, MSI-H/dMMR solid tumors with progression on prior treatment with no satisfactory alternative treatment options (8). This was the FDA's first tissue/site agnostic approval, and was based on the combined results of five multi-cohort, single-arm clinical trials evaluating pembrolizumab in patients with metastatic or unresectable solid tumors who had received a median of two prior lines of therapy. Of 149 patients with MSI-H/dMMR cancers across all five studies, there were 59 responders for an objective response rate (ORR) of $36.9 \%$ and a complete response rate of $7 \%$ (9-11). Of the five studies included in the FDA approval summary, the study by Le $e t$ al. Science 2017 was the first to demonstrate a benefit of PD-1 blockade in pancreatic cancers. Eight of the 86 patients included in the study had pancreatic cancer, and the ORR among pancreatic cancer patients was $62 \%$ (2 patients had complete responses, 3 patients had partial responses, 1 patient had stable disease, and 2 patients were not evaluable) (9).

Subsequent reports on PD-1 inhibition in MSI-H/ dMMR solid tumors have demonstrated lower response rates in pancreatic cancer. KEYNOTE-158 was a nonrandomized, open-label, multicenter, multi-cohort phase II study evaluating pembrolizumab in a variety of tumor types, including a specific cohort of non-colorectal MSI-H/dMMR solid tumors previously treated with standard therapy. Efficacy results from the non-colorectal MSI-H/dMMR solid tumor cohort of KEYNOTE-158 were recently published (12). Patients were treated with pembrolizumab $200 \mathrm{mg}$ once every 3 weeks for 35 cycles or until disease progression, treatment-limiting toxicity, intercurrent illness, or patient/investigator decision to withdraw from the study. The ORR among 233 patients across 27 tumor types was $34.4 \%$. While these pooled response rates to pembrolizumab in pretreated patients with MSI-H/dMMR cancers were encouraging-the response rate in the subset of patients with MSI-H/dMMR pancreatic cancers was not as robust. Of 22 patients with pancreatic cancer included in the study, 3 partial responses and 1 complete response to pembrolizumab were observed (ORR 18.2\%). The median overall survival was 4.0 months in the pancreatic cancer subgroup, although it should be noted that the median duration of response was 13.4 months.

These results from small numbers of patients with MSI-H/dMMR pancreatic cancers are difficult to generalize as the incidence of mismatch repair deficiency in PDAC has been reported to range from $0.8 \%$ to $2 \%(9,13-15)$. PDAC has long been thought of as an immunologically "cold" malignancy, with multiple barriers to effective immunotherapy in the PDAC tumor microenvironment (16). Attempting to overcome these barriers by utilizing a combination of anti-PD-1/anti-programmed death ligand-1 (PD-L1) checkpoint inhibitors with other immune and targeted therapies is a logical next step.

O'Reilly et al. recently published negative results from the first phase II randomized clinical trial evaluating dual immune checkpoint blockade in patients with advanced PDAC (17). This was a multicenter, two-part, phase II randomized clinical trial of the PD-L1 antibody durvalumab with or without the anti-cytotoxic T-lymphocyte antigen-4 (CTLA-4) antibody tremelimumab in patients with recurrent or metastatic PDAC. Study participants had received one prior gemcitabine-based or fluorouracilbased chemotherapy regimen. Sixty-five patients were randomized to part A of the study. The ORR was 3.1\% for patients treated with the combination of durvalumab and tremelimumab and $0 \%$ for patients treated with durvalumab monotherapy. As part A did not meet the prespecified threshold for study expansion (10\% ORR in either treatment arm), the study was closed. PD-L1 expression was evaluated, however the low number of responses was inadequate for analysis of PD-L1 and clinical response association. This study highlights some of the challenges of effective immunotherapy in PDAC.

Effective immunotherapy for PDAC that is not 
Table 1 Selected immunotherapy trials for pancreatic adenocarcinoma

\begin{tabular}{|c|c|c|c|c|}
\hline Study name & Phase & Immunotherapy & Status & $\begin{array}{l}\text { ClinicalTrials.gov } \\
\text { identifier }\end{array}$ \\
\hline Car T cell immunotherapy for pancreatic cancer (20) & I & huCAR-T meso cells & Recruiting & NCT03323944 \\
\hline $\begin{array}{l}\text { LCAR-C182A cells in the treatment of advanced gastric cancer and } \\
\text { pancreatic ductal adenocarcinoma (21) }\end{array}$ & I & LCAR-C182A cells & Recruiting & NCT03890198 \\
\hline Autologous T-cells in patients with metastatic pancreatic cancer (22) & I & CAR-T meso cells & Recruiting & NCT03638193 \\
\hline $\begin{array}{l}\text { Th-1 dendritic cell immunotherapy plus standard chemotherapy for } \\
\text { pancreatic adenocarcinoma (DECIST) (23) }\end{array}$ & I & $\begin{array}{l}\text { Autologous dendritic } \\
\text { cell vaccine }\end{array}$ & Not yet recruiting & NCT04157127 \\
\hline $\begin{array}{l}\text { Nivolumab and ipilimumab and radiation therapy in MSS and MSI } \\
\text { high colorectal and pancreatic cancer (24) }\end{array}$ & II & $\begin{array}{l}\text { Nivolumab and } \\
\text { ipilimumab }\end{array}$ & Recruiting & NCT03104439 \\
\hline $\begin{array}{l}\text { Study of anti-CEA CAR-T + chemotherapy vs chemotherapy alone } \\
\text { in patients With CEA+pancreatic cancer \& liver metastases (25) }\end{array}$ & II & Anti-CEA CAR-T cells & Not yet recruiting & NCT04037241 \\
\hline Plerixafor and cemiplimab in metastatic pancreatic cancer (26) & II & Cemiplimab & Not yet recruiting & NCT04177810 \\
\hline
\end{tabular}

MSI-H/dMMR remains elusive and is an area of ongoing clinical research. Studies investigating PD-1/PD-L1 inhibition in combination with other therapies in PDAC are ongoing (16). A recently published phase I study of nivolumab and the anti-CC chemokine receptor 4 antibody mogamulizumab in patients with advanced solid tumors included 15 patients with PDAC (18). The authors reported 1 confirmed partial response and 2 unconfirmed partial responses in patients with PDAC. These results should be interpreted within the limitations of a phase I study. Novel therapies in preclinical development are investigating the use of CAR T-cell therapy against PDAC (19). Selected immunotherapy clinical trials for pancreatic adenocarcinoma are listed in Table 1. We will await the results of these ongoing studies that offer hope for successful pancreatic cancer immunotherapy.

\section{Acknowledgments}

Funding: None.

\section{Footnote}

Provenance and Peer Review: This article was commissioned by the Guest Editor (Khaldoun Almhanna) for the series "GI malignancies and Immunotherapy" published in Translational Gastroenterology and Hepatology. The article has undergone external peer review.

Conflicts of Interest: Both authors have completed the
ICMJE uniform disclosure form (available at http://dx.doi. org/10.21037/tgh.2020.04.03). KA served as the unpaid Guest Editor of the series and has a consulting agreement with Merck. JB has no conflicts of interest to declare. The series "GI malignancies and Immunotherapy" was commissioned by the editorial office without any funding or sponsorship.

Ethical Statement: The authors are accountable for all aspects of the work in ensuring that questions related to the accuracy or integrity of any part of the work are appropriately investigated and resolved.

Open Access Statement: This is an Open Access article distributed in accordance with the Creative Commons Attribution-NonCommercial-NoDerivs 4.0 International License (CC BY-NC-ND 4.0), which permits the noncommercial replication and distribution of the article with the strict proviso that no changes or edits are made and the original work is properly cited (including links to both the formal publication through the relevant DOI and the license). See: https://creativecommons.org/licenses/by-nc-nd/4.0/.

\section{References}

1. Balli D, Rech AJ, Stanger BZ, et al. Immune Cytolytic Activity Stratifies Molecular Subsets of Human Pancreatic Cancer. Clin Cancer Res 2017;23:3129-38.

2. Vonderheide RH, Bayne LJ. Inflammatory networks and immune surveillance of pancreatic carcinoma. Curr Opin 
Immunol 2013;25:200-5.

3. Bernhardt SL, Gjertsen MK, Trachsel S, et al. Telomerase peptide vaccination of patients with non-resectable pancreatic cancer: A dose escalating phase I/II study. Br J Cancer 2006;95:1474-82.

4. Middleton G, Silcocks P, Cox T, et al. Gemcitabine and capecitabine with or without telomerase peptide vaccine GV1001 in patients with locally advanced or metastatic pancreatic cancer (TeloVac): an open-label, randomised, phase 3 trial. Lancet Oncol 2014;15:829-40.

5. NewLink Genetics announces results from phase 3 IMPRESS trial of algenpantucel-L for patients with resected pancreatic cancer. Ames, IA: NewLink Genetics, May 9, 2016.

6. Le DT, Wang-Gillam A, Picozzi V, et al. Safety and survival with GVAX pancreas prime and Listeria Monocytogenes-expressing mesothelin (CRS-207) boost vaccines for metastatic pancreatic cancer. J Clin Oncol 2015;33:1325-33.

7. Le DT, Picozzi VJ, Ko AH, et al. Results from a Phase IIb, Randomized, Multicenter Study of GVAX Pancreas and CRS-207 Compared with Chemotherapy in Adults with Previously Treated Metastatic Pancreatic Adenocarcinoma (ECLIPSE Study). Clin Cancer Res 2019;25:5493-502.

8. FDA grants accelerated approval to pembrolizumab for first tissue/site agnostic indication. Silver Spring (MD): U.S. Food and Drug Administration, May 30, 2017.

9. Le DT, Durham JN, Smith KN, et al. Mismatch repair deficiency predicts response of solid tumors to PD-1 blockade. Science 2017;357:409-13.

10. Le DT, Uram JN, Wang H, et al. PD-1 Blockade in Tumors with Mismatch-Repair Deficiency. N Engl J Med 2015;372:2509-20.

11. Marcus L, Lemery SJ, Keegan P, et al. FDA Approval Summary: Pembrolizumab for the Treatment of Microsatellite Instability-High Solid Tumors. Clin Cancer Res 2019;25:3753-8.

12. Marabelle A, Le DT, Ascierto PA, et al. Efficacy of Pembrolizumab in Patients With Noncolorectal High Microsatellite Instability/Mismatch Repair-Deficient Cancer: Results From the Phase II KEYNOTE-158 Study. J Clin Oncol 2020;38:1-10.

13. Hu ZI, Shia J, Stadler ZK, et al. Evaluating Mismatch Repair Deficiency in Pancreatic Adenocarcinoma: Challenges and Recommendations. Clin Cancer Res 2018;24:1326-36.

14. Humphris JL, Patch AM, Nones K, et al. Hypermutation In Pancreatic Cancer. Gastroenterology 2017;152:68-74.e2.
15. Lupinacci RM, Goloudina A, Buhard O, et al. Prevalence of Microsatellite Instability in Intraductal Papillary Mucinous Neoplasms of the Pancreas. Gastroenterology 2018;154:1061-5.

16. Balachandran VP, Beatty GL, Dougan SK. Broadening the Impact of Immunotherapy to Pancreatic Cancer: Challenges and Opportunities. Gastroenterology 2019;156:2056-72.

17. O'Reilly EM, Oh DY, Dhani N, et al. Durvalumab With or Without Tremelimumab for Patients With Metastatic Pancreatic Ductal Adenocarcinoma: A Phase 2 Randomized Clinical Trial. JAMA Oncol 2019. [Epub ahead of print].

18. Doi T, Muro K, Ishii H, et al. A Phase I Study of the AntiCC Chemokine Receptor 4 Antibody, Mogamulizumab, in Combination with Nivolumab in Patients with Advanced or Metastatic Solid Tumors. Clin Cancer Res 2019;25:6614-22.

19. Yazdanifar M, Zhou R, Grover P, et al. Overcoming Immunological Resistance Enhances the Efficacy of A Novel Anti-tMUC1-CAR T Cell Treatment against Pancreatic Ductal Adenocarcinoma. Cells 2019. doi: 10.3390/cells8091070.

20. CAR T Cell Immunotherapy for Pancreatic Cancer. ClinicalTrials.gov Identifier: NCT03323944. Available online: https://clinicaltrials.gov/ct2/show/NCT03323944. Updated January 18, 2020. Accessed March 14, 2020.

21. A Phase 1 Study of LCAR-C182A Cells in the Treatment of Advanced Gastric Cancer and Pancreatic Ductal Adenocarcinoma. ClinicalTrials.gov Identifier: NCT03890198. Available online: https://clinicaltrials.gov/ ct2/show/NCT03890198. Updated November 4, 2019. Accessed March 14, 2020.

22. Study of Autologous T-cells in Patients With Metastatic Pancreatic Cancer. ClinicalTrials.gov Identifier: NCT03638193. Available online: https://clinicaltrials.gov/ ct2/show/NCT03638193. Updated December 27, 2018. Accessed March 14, 2020.

23. Th-1 Dendritic Cell Immunotherapy Plus Standard Chemotherapy for Pancreatic Adenocarcinoma (DECIST). ClinicalTrials.gov Identifier: NCT04157127. Available online: https://clinicaltrials.gov/ct2/show/NCT04157127. Updated March 10, 2020. Accessed March 14, 2020.

24. Nivolumab and Ipilimumab and Radiation Therapy in MSS and MSI High Colorectal and Pancreatic Cancer. ClinicalTrials.gov Identifier: NCT03104439. Available online: https://clinicaltrials.gov/ct2/show/NCT03104439. Updated June 27, 2019. Accessed March 14, 2020. 
25. Study of Anti-CEA CAR-T + Chemotherapy VS Chemotherapy Alone in Patients With CEA+Pancreatic Cancer \& Liver Metastases. ClinicalTrials.gov Identifier: NCT04037241. Available online: https://clinicaltrials. gov/ct2/show/NCT04037241. Updated August 5, 2019. Accessed March 14, 2020.

doi: $10.21037 / \operatorname{tgh} .2020 .04 .03$

Cite this article as: Bian J, Almhanna K. Pancreatic cancer and immune checkpoint inhibitors-still a long way to go. Transl Gastroenterol Hepatol 2021;6:6.
26. Plerixafor and Cemiplimab in Metastatic Pancreatic Cancer. ClinicalTrials.gov Identifier: NCT04177810. Available online: https://clinicaltrials.gov/ct2/show/ NCT04177810. Updated February 13, 2020. Accessed March 14, 2020. 